\title{
Abordagem dos temas Bioproteção e Bioterrorismo na Educação: Proposta de Curso
}

\section{de Extensão}

\author{
Approach to Bioprotection and Bioterrorism themes in Education: Extension Course Proposal \\ Enfoque de los Temas de Bioprotección y Bioterrorismo en la Educación: Propuesta de Curso de \\ Extensión
}

Recebido: 21/12/2021 | Revisado: 30/12/2021 | Aceito: 25/01/2022 | Publicado: 06/02/2022

Lidiane Coelho Berbert

ORCID: https://orcid.org/0000-0002-4882-176X Fundação Centro Universitário Estadual da Zona Oeste, Brasil E-mail: lidy_berbert@hotmail.com

Vinicius Riberio Flores

ORCID: https://orcid.org/0000-0002-0682-7995

Fundação Centro Universitário Estadual da Zona Oeste, Brasil

E-mail: viniciusflores42@gmail.com

Ida Carolina Neves Direito

ORCID: https://orcid.org/0000-0002-3455-8091

Fundação Centro Universitário Estadual da Zona Oeste, Brasil

E-mail: idacarolinadireito@gmail.com

Alexander Machado Cardoso

ORCID: https://orcid.org/0000-0003-2974-0232

Fundação Centro Universitário Estadual da Zona Oeste, Brasil

E-mail: amcardosopf@yahoo.com.br

\begin{abstract}
Resumo
A Natureza é fonte de recursos para a sobrevivência do homem desde sempre, porém o ser humano também viu nela uma oportunidade de utilização com outras finalidades, passando a explorar então o potencial patogênico de diversos organismos a fim de tirar vantagens econômicas e em conflitos. Dessa forma, foram necessárias medidas que visassem controlar o acesso e uso desses organismos, a bioproteção. Assim como muitos outros assuntos, a bioproteção e o bioterrorismo são temáticas de difícil abordagem. Assim, se fazem necessárias técnicas que viabilizem a aproximação e melhorem a abordagem do tema junto ao estudante. O objetivo desse trabalho foi fazer através de revisão na literatura, um levantamento de atividades e ferramentas que possam ser utilizadas como meios de aproximação e divulgação científica, dentro da temática bioproteção e bioterrorismo, propondo um curso de extensão que desperte o interesse dos alunos.
\end{abstract}

Palavras-chave: Educação; Ensino; Cursos; Práticas Pedagógicas.

\begin{abstract}
Nature has always been a source of resources for human survival, but they also being saw in it an opportunity to use it for other purposes, starting to explore the pathogenic potential of many organisms in order to take economic and conflict advantages. Thus, measures were needed to control the access and use of these organisms, bioprotection. Such as many other issues, bioterrorism and bioprotection are difficult-to-approach topics. Thus, techniques are needed that make closer feasible and improve the approach to the topic with the students. The aim of this work was to carry out a literature review, a survey of activities and tools that can be used as means of approximation and scientific dissemination, within the theme of bioprotection and bioterrorism, proposing an extension course that arouses the interest of students.
\end{abstract}

Keywords: Education; Teaching; Courses; Pedagogical Practices.

\section{Resumen}

La naturaleza siempre ha sido fuente de recursos para la supervivencia humana, pero los seres humanos también vieron en ella la oportunidad de utilizarla para otros fines, comenzando a explorar el potencial patógeno de diversos organismos con el fin de tomar ventajas económicas y en conflictos. Por tanto, se necesitaban medidas para controlar el acceso y uso de estos organismos, bioprotección. Como muchos otros temas, el bioterrorismo y la bioprotección son temas difíciles de abordar. Por tanto, se necesitan técnicas que viabilicen la aproximación y mejoren la aproximación al tema con los estudiantes. El objetivo de este trabajo fue realizar a través de una revisión en la literatura, un relevamiento de actividades y herramientas que puedan ser utilizadas como medios de aproximación y 
divulgación científica, dentro de la temática de bioprotección y bioterrorismo, proponiendo un curso de extension que despierte el interés de los estudiantes.

Palabras clave: Educación; Docencia; Cursos; Prácticas pedagógicas.

\section{Introdução}

Está na Natureza, em sua biodiversidade, um dos pilares centrais para o desenvolvimento do ser humano. Fornecedora de matéria-prima desde a antiguidade, não demorou até que o homem encontrasse nela também armas a serem utilizadas em conflitos com os mais diferentes propósitos. Dentre alguns registros desse uso ilícito do conhecimento e da Natureza, destacam-se a utilização do potencial patogênico de organismos, como por exemplo: o uso de animais mortos pelos romanos para contaminar o suprimento de água dos inimigos; o uso dos corpos de vítimas da peste negra em batalhas; a distribuição de cobertores contaminados com varíola aos índios pelo exército britânico; utilização de salada contaminada com bactérias para manipular o resultado das eleições locais e mais recentemente, o envio de antrax por correio a imprensa e escritórios do governo americano (Cristopher et al., 1997; Lim et al., 2005; Silva, 2012).

Os relatos de casos, ainda que mais isolados, continuam nos dias atuais, e esse instinto não se modificou com o tempo. Pode-se dizer que os avanços e revoluções científicas e tecnológicas na área biológica podem se tornar um fator de risco, se não houver mudanças de hábitos, postura e índole por parte do ser humano (Augusto, 2012).

Diante disso, foi necessário que órgãos competentes de todo o mundo como a ONU (Organização das Nações Unidas) e a OMS (Organização Mundial de Saúde) se reunissem para buscar uma forma de controlar o acesso e uso de organismos como armas biológicas, o que envolve questões éticas, acordos governamentais, mas especialmente, a proteção e controle no acesso e uso de microrganismos (Silva, 2012; Augusto, 2012). Assim nasceu a bioproteção, que envolve desde medidas de segurança pessoais, individuais e institucionais que podem ser de característica física ou administrativa a fim de evitar perda, roubo, desvio, liberação (intencional ou não) e uso indevido de vírus, organismos vivos, como patógenos, bem como toxinas ou partes dos mesmos (Silva, 2012).

A bioproteção tem ganhado cada vez mais notoriedade, principalmente no que diz respeito a bancos de cepas, pois neles estão estocados microrganismos que já causaram grandes crises mundiais como, por exemplo, amostras do vírus Orthopoxvirus variolae, causador da varíola, doença considerada erradicada desde 1980 (Levi \& Kallas, 2002). Outro exemplo de microrganismo altamente patogênico que se encontra em bancos de cepas é a Yersinia pestis, causadora da peste negra que assolou a Europa em meados do século XIV levando a morte de 200 milhões de pessoas (Rezende, 2009).

Esses organismos são considerados verdadeiras armas biológicas e têm fácil disseminação, alta taxa de mortalidade, produção fácil e de baixo custo, além de serem capazes de se manterem e replicarem, se autoperpertuando, podendo gerar grande pânico na população (Rezende, 2009; Silva, 2012). Dentro da bioproteção existem três categorias de risco de acordo com o objetivo e o grau de envolvimento das partes: 1) guerra biológica, com objetivos de curto a longo prazo, envolve nações, investidas militares e atentados em massa; 2) bioterrorismo, envolve grupos (religiosos, políticos e ideológicos) com objetivos pontuais, mais direcionados e a curto prazo; 3 ) os bioataques com objetivos de curto prazo com ações individuais, como por exemplo, o uso de pragas para destruir plantações de concorrentes ou provocar doenças em rebanhos, criações de peixes e crustáceos (Cardoso \& Cardoso, 2011; Silva, 2012).

Dentro dessa questão de proteção e controle quanto ao uso de organismos vivos, estão aumentando também as iniciativas de combate a biopirataria, que nada mais é do que a exploração e utilização de recursos naturais e genéticos, de qualquer natureza, de forma ilegal, o que inclui também questões relacionadas ao conhecimento popular (Saccaro, 2011; Felicio, 2019). Esse processo tem uma aplicação ampla, sendo utilizado para referência ao contrabando de aves e outros animais silvestres e plantas, principalmente se tratando de plantas com potencial medicinal e/ou terapêutico (Rocha, 2019).

Diversos sistemas foram criados para tentar monitorar o que está sendo feito com a biodiversidade estudada, por 
exemplo, a Lei da Biodiversidade, as autorizações de coleta em áreas protegidas ou ao ICMBio (Instituto Chico Mendes de Conservação da Biodiversidade) e o cadastro de pesquisa junto CGEN (Conselho de Gestão do Patrimônio Genético) (Rocha, 2019). O que por muitos é visto como burocracia, na verdade são formas de proteção da biodiversidade e de monitoramento do que está sendo feito, a fim de tentar evitar, por exemplo, o uso indevido de organismos vivos e seus compostos. Neste trabalho, a partir de uma revisão sistemática da literatura e análises sobre o ensino da bioproteção e bioterrorismo, uma proposta de curso de extensão foi idealizada para abordar todas essas questões de forma intuitiva e que desperte o interesse do público em geral.

\section{Metodologia}

A metodologia utilizada na pesquisa resumiu-se em uma abordagem descritiva de natureza qualitativa (Pereira et al., 2018). Este trabalho foi elaborado através de levantamento da literatura em diversos sites acadêmicos como Google Acadêmico, SciELO, Science Direct, utilizando palavras-chaves como bioproteção, bioterrorismo, divulgação científica, educação, biossegurança, biosseguridade, bioataques, sendo estes pesquisados também em inglês.

\section{Resultados e Discussão}

Diante de questões tão sérias e de alto risco envolvendo microrganimos e descobertas científicas, como manipulação genética e de outras moléculas com potencial de uso maléfico, é difícil pensar como abordar tais questões dentro das escolas, porém um primeiro ponto é justamente mostrar o outro lado da história. Em 2016, Berbert e colaboradores destacaram a importância de esclarecer a população que microrganismos podem ser benéficos, ressaltando que estes não são só causadores de doenças como muitos pensam, afinal, é o que lhes é mostrado, e que mesmo muito presente no dia-a-dia das pessoas, passam despercebidos ou vistos sempre como vilões. Muitos destes organismos são utilizados desde o início dos tempos, afim de beneficiar o ser humano, como por exemplo, na fermentação de pães, vinhos, cervejas, produção de antibióticos e recuperação de áreas poluídas.

Esta apresentação ao público é importante para compreenderem que assim como existem grupos que utilizam os microrganismos com a finalidade de prejudicar o próximo fisicamente ou economicamente, como a utilização do $B$. anthracis para causar pânico e o óbito de alvos específicos, bemo como a utilização de microrganismos que causam doenças em plantações como o ocorrido nas plantações de seringueira e ervas utilizados em chás no Sri Lanka pelos militantes tâmeis (Gori \& Tomar, 2020), também existem grupos que buscam a utilização desses microrganismos para fins benéficos, além dos exemplos clássicos já citados, existem grupos de pesquisa que buscam utilizar microrganismos para a biorremediação de solos e inoculantes para utilizar na agricultura (Sbano et al., 2020), ou se utilizam de microrganismos para controle biológico de pragas e vetores de doenças, em consórcio e/ou alternativa à utilização de pesticidas químicos (Maciel et al., 2022), ou ainda, baseiam suas pesquisas em toxinas e subprodutos desses microrganismos com finalidade médica, estética, entre outros, como é o caso do uso da toxina botulínica (Gouveia et al., 2020).

Como colocado por Silva (2012) e Augusto (2012) é importante sempre destacar a dualidade das coisas, os microrganismos podem ser utilizados para o bem ou para o mal (uso dual), tudo vai depender do uso que é feito, o propósito atribuído. Em outras palavras, será principalmente, uma questão de caráter. Dessa forma, a formação de cidadãos com princípios éticos cada vez mais fortes e estruturados, reduziria a longo prazo os perigos e riscos envolvendo o uso de organismos de forma indevida (Tapajós, 2011; Da Silva, 2012; Augusto, 2012; Costa, 2019).

$\mathrm{O}$ ensino de ciências sempre enfrentou e enfrenta dificuldades, seja pela infinidade de conceitos que engloba, mudanças decorrentes de novas descobertas, ou por questões estruturais ou pedagógicas (Portela, 2020). No que diz respeito a bioproteção e o bioterrorismo, assim como muitas outras temáticas dentro do meio científico, não é diferente, pois são de 
dificil abordagem, devido a seriedade e problemáticas do assunto, além de sua complexidade. Porém, de um modo geral, é preciso, incentivar nas crianças e jovens a curiosidade científica e aproximá-los da pesquisa e de todo o universo científico, seja através de jogos, atividades interativas, oficinas, visitas a laboratórios (Lab Tour/Lab Day), como as descritas por Direito e colaboradores (2018), atividades que lhes permitam não só observar ou assistir, mas principalmente, fazer, interagir e despertar a curiosidade, o senso crítico, o pensar e viver ciência. Ainda nas atividades descritas pelos autores, era possível a observação de cepas ao microscópio, realização de bioprospecção de microrganismos do solo, observação de células e bactérias da boca, a produção e consumo de iogurte produzido pelos próprios participantes. Durante a pandemia do novo coronavírus, com a necessidade do distanciamento social, incentivou-se uma modalidade de visitação que antes não era dada a devida atenção, a visitação virtual de diversos espaços, como por exemplo museus, exposições e até laboratórios que permitiu essa aproximação, levando todos esses ambientes em $360^{\circ}$ às casas do público (Clemonz, 2019).

Outra ferramenta muito importante, que vem auxiliando o processo de ensino-aprendizagem e aproximação da população com a ciência é a utilização de elementos da cultura, como personagens de filmes, séries, desenhos e jogos (Miller \& Metz, 2014). Dentro dessa categoria, uma possibilidade para abordagem da bioproteção e bioterrorismo é o jogo Plague Inc (https://www.ndemiccreations.com/en/22-plague-inc), onde o objetivo do jogo é criar uma praga mortal a nível mundial. O próprio jogo traz questões relacionadas a saúde pública, levando o jogador a reflexão sobre o assunto, podendo ser uma excelente porta de entrada para abordar bioproteção com o público em geral, em especial crianças e adolescentes.

A fim de estimular os estudantes para o debate destes temas, sugerimos a elaboração de um curso de extensão que teria uma carga horária total de 15h. Alguns tópicos foram pensados como ementa, conforme Quadro 1 a seguir:

Quadro 1 - Ementa do curso de extensão em bioproteção e bioterrorismo.

\begin{tabular}{|c|c|}
\hline \multicolumn{2}{|c|}{ EMENTA } \\
\hline $\begin{array}{c}\text { 1. Introdução à bioproteção e bioterrorismo } \\
\text { 2. Conceitos gerais, histórico, importância e } \\
\text { legislação }\end{array}$ & $\begin{array}{c}\text { 4. Manuseio, armazenamento e descarte de agentes } \\
\text { biológicos }\end{array}$ \\
\hline $\begin{array}{c}\text { 3. Noções de segurança biológica em laboratório } \\
\text { no Mundo }\end{array}$ & $\begin{array}{c}\text { 6. O futuro da bioproteção e bioterrorismo no Brasil e } \\
\text { no Mundo }\end{array}$ \\
\hline
\end{tabular}

Fonte: Autores.

Os recursos didáticos que podem ser utilizados incluem: aulas práticas, discussão de artigos científicos, utilização de ferramentas online, jogos e vídeos. Segue abaixo modelo de cronograma (Quadro 2), divido em 3 blocos de 5 horas cada: 
Quadro 2 - Cronograma das atividades.

\begin{tabular}{|c|c|c|c|}
\hline . & & Ferramenta & Objetivo \\
\hline \multirow{4}{*}{ Bloco 1} & \multirow{4}{*}{ Ambientalização } & Formulário I & $\begin{array}{l}\text { Verificar o conhecimento inicial do grupo a } \\
\text { respeito do tema }\end{array}$ \\
\hline & & Dinâmica & $\begin{array}{l}\text { Criação de nuvens de palavras e outros } \\
\text { recursos digitais criados ao vivo junto dos } \\
\text { participantes sobre a temática }\end{array}$ \\
\hline & & Jogo & \begin{tabular}{|l} 
Após conversa e ambientalização, os \\
participantes serão convidados a uma partida \\
de jogos como Plague Inc e Pandemic
\end{tabular} \\
\hline & & Debate & $\begin{array}{l}\text { Bate-papo/debate sobre o tema baseado na } \\
\text { experiência desse primeiro bloco de atividades }\end{array}$ \\
\hline \multirow{4}{*}{ Bloco 2} & \multirow{4}{*}{ Apresentação do conteúdo } & Histórico & \multirow{4}{*}{$\begin{array}{l}\text { Apresentação do conteúdo por recursos } \\
\text { audio-visuais, abordando breve histórico, } \\
\text { algumas } \\
\text { acontecimentos mais recentes e debate com } \\
\text { base nas atividades dos blocos }\end{array}$} \\
\hline & & Definições & \\
\hline & & $\begin{array}{l}\text { Acontecimentos } \\
\text { recentes }\end{array}$ & \\
\hline & & Debate & \\
\hline \multirow{3}{*}{ Bloco 3} & \multirow{3}{*}{ Uso dual + encerramento } & $\begin{array}{l}\text { Utilizações benéfica } \\
\text { de microrganismos }\end{array}$ & $\begin{array}{l}\text { Apresentação da utilização dos } \\
\text { microrganismos e da biodiversidade para fins } \\
\text { benéficos, abordado também a importância da } \\
\text { conservação de recursos }\end{array}$ \\
\hline & & LabTour & $\begin{array}{l}\text { Visitação guiada do grupo a um laboratório } \\
\text { de pesquisa }\end{array}$ \\
\hline & & Formulário II & $\begin{array}{l}\text { Formulário de saída, para analisar os } \\
\text { resultados das atividades realizadas }\end{array}$ \\
\hline
\end{tabular}

Fonte: Autores.

\section{Considerações Finais}

A aproximação da ciência com a sociedade, muitas vezes não é um processo fácil, mas é a chave para diminuir e até mesmo solucionar muitos problemas enfrentados atualmente, não só no que diz respeito aos temas de bioproteção, biopirataria, bioterrorismo, mas também no combate de fakenews e movimentos anti-ciência. Diante do cenário negacionista que pôde ser observado durante a pandemia de COVID-19, ficou cada vez mais clara a importância da Educação e da Ciência, reforçando a necessidade de uma boa aproximação e comunicação da ciência. Infelizmente, a sociedade ainda nos dias atuais tem uma visão distante de tudo que a ciência vem produzindo e viabilizando. A mudança no cenário que nos foi apresentado de forma mais crítica nos últimos dois anos (2020 e 2021) só será possível através do conhecimento, da derrubada dos muros que ainda existem entre a ciência e a sociedade. O presente trabalho propõe um curso de extensão mais atrativo sobre bioproteção e bioterrorismo, alertando sobre a necessidade de incentivar nas crianças e jovens a curiosidade científica e aproximá-los da pesquisa e de todo o universo científico, sejam através de jogos, atividades interativas, oficinas, visitas a laboratórios virtuais e presenciais. Artigos ou trabalhos futuros sobre essa metodologia e o impacto no aprendizado desses temas pelos alunos se fazem necessários.

\section{Agradecimentos}

Agradecemos ao Conselho Nacional de Desenvolvimento Científico e Tecnológico (CNPq), Coordenação de Aperfeiçoamento de Pessoal de Nível Superior (CAPES) e Fundação Carlos Chagas Filho de Apoio à Pesquisa do Estado do Rio de Janeiro (FAPERJ) pelo apoio financeiro e à equipe da UEZO pelas discussões. 


\section{Referências}

Augusto, L. G. da S. (2012). Reflexão crítica sobre a invisibilidade da biossegurança e da biosseguridade. Ciência \& Saúde Coletiva, 17 (2), 293 - 294.

Cardoso, D. R., \& Cardoso, T. A. O. (2011). Bioterrorismo: dados de uma história recente de riscos e incertezas. Ciência \& Saúde Coletiva, 16 (1), 821-830.

Christopher, G., T., Ciesklak, J., Pavlin, E., \& Eitzen. (1997). BiologicaL warfare: a historical perspective. JAMA $278,412-417$.

Clemons, T. D., Fouché, L., Rummey, C., Lopez, R. E., \& Spagnoli, D. (2019) Introducing the first year laboratory to undergraduate chemistry students with an interactive 360 experience. J. Chem. Educ., 96 (7), 1491-1496.

Costa, F. (2019). O papel da educação na formação da personalidade. Revista Científica Multidisciplinar Núcleo do Conhecimento, 03 , 82-97.

Direito, I. C. N. et al. (2018). Educação e integração: Popularização da ciência e Tecnologia promovendo o desenvolvimento regional. In: Manchope, E.C.P. et al. Interiorização do Ensino superior: protagonismo das universidades estaduais e municipais no desenvolvimento regional. Cascavel, PR: EDUNIOESTE.

Felicio, G. M. B. (2019). Criminalização da biopirataria: dogmática e necessidade. Dissertação. Direito. UNESP. 112.

Gori, S. \& Tomar, A. S. (2020). Bioterrorism \& Biodefense: An Environmental and Public Health Preparedness. Rupkatha Journal on Interdisciplinary Studies in Humanities (ISSN 0975-2935). DOI: https://dx.doi.org/10.21659/rupkatha.v12n2.13

Gouveia, B. N. et al. (2020). O uso da toxina botulínica em procedimentos estéticos. Revista Brasileira Militar de Ciências. 6 (16), $56-63$.

Levi, G.C., \& Kallas, E.G. (2002) Varíola, sua prevenção vacinal e ameaça como agente de bioterrorismo. Rev. Assoc. Med. Bras., 48 (4), $357-362$.

Lim, D. V, Simpson, J. M., Kearns, E. A., \& Kramer, M. F. (2005). Current and Developing Technologies for Monitoring Agents of Bioterrorism and Biowarfare. Clinical Microbiology Reviews, 583-607.

Maciel, R. M. A. et al. (2022). Mixture compatibility of Anticarsia gemmatalis nucleopolyhedrovirus (AgMNPV) with pesticides used in soybean. Ciência Rural [online], 52 (2). https://doi.org/10.1590/0103-8478cr20210027.

Miller, C. J., \& Metz, M. J. (2014). A comparison of professional-level faculty and student perceptions of active learning: its current use, effectiveness, and barriers. Advances in Physiology Education, 38 (3).

Pereira, A. S., Shitsuka, D. M., Parreira, F. J.: Shitsuka, R. (2018). Metodologia da pesquisa científica. UFSM. https://www.ufsm.br/app/uploads/sites/358/2019/02/Metodologia-da-Pesquisa-Cientifica_final.pdf

Rezende, J. M. (2009). As grandes epidemias da história. In: À sombra do plátano: crônicas de história da medicina. Editora Unifesp, 73-82.

Rocha, M.C.A. Biopirataria das plantas medicinais enquanto apropriação dos conhecimentos tradicionais da Amazônia Brasileira. Dissertação de mestrado. Direito. UFSM. 183. 2019.

Saccaro JR, N.L. (2011). A regulamentação de acesso a recursos genéticos e repartição de benefícios: disputas dentro e fora do Brasil. Ambient. Soc., 14 (1), 229-244.

Sbano, A., Succar, J. B., Ferreira, J. V. R., Berbert, L. C., Flores, V. R., Cavalcante, J. J. V., Albano, R. M., Direito, I. C. N., \& Cardoso, A. M. (2020). Data on the Draft Genome Sequence of Bacillus sp. Strain AN2 Isolated from Agricultural Soil in Brazil. Asian Journal of Biotechnology and Bioresource Technology, 6(1), 11-13. https://doi.org/10.9734/ajb2t/2020/v6i130071

Silva, M. (2012). Bioproteção - Conceitos, Requisitos do Guia de Boas práticas da OCDE e ligação com os requisitos da ABNT http://www.inmetro.gov.br/credenciamento/pdf/1_bioprotecao.pdf

Portela, A. P. P., \& Barreto, L. (2020). Construção de Material Didático sobre Controle Biológico: um Olhar para o Ensino de Ciências. Revista Prática Docente, 5(3), 1944-1963. https://doi.org/10.23926/RPD.2526-2149.2020.v5.n3.p1944-1963.id872

Tapajós, A. C. (2011). Bioética e Armas Biológicas No Contexto Internacional. Tese de doutorado. Ciências da Saúde. Universidade de Brasília. Brasília. 469. 